\title{
Near-Infrared Reflectance Spectroscopy (NIRs) for Determination of Tryptophan Content in Quality Protein Maize (QPM)
}

\author{
Legesse Shiferaw, Girmay Tsegay, Gelila Asamenew \\ Ethiopian Institute of Agricultural Research, Agricultural Quality Research Laboratory, Adiss Abeba, Ethiopia \\ Email address: \\ legesse13@yahoo.com (L. Shiferaw), girmaytg7@gmail.com (G. Tsegay), gelilase@yahoo.com (G. Asamenew)
}

To cite this article:

Legesse Shiferaw, Girmay Tsegay, Gelila Asamenew. Near-Infrared Reflectance Spectroscopy (NIRs) for Determination of Tryptophan Content in Quality Protein Maize (QPM). Science Journal of Analytical Chemistry. Vol. 5, No. 1, 2017, pp. 8-11. doi: $10.11648 /$ j.sjac.20170501.12

Received: December 25, 2016; Accepted: January 5, 2017; Published: February 10, 2017

\begin{abstract}
Quality protein maize (QPM) has approximately twice tryptophan (Trp) and lysine (Lys) concentrations in protein compared to normal maize. Because several genetic systems control the protein quality of QPM, it is essential to regularly monitor Trp and/or Lys in QPM breeding programs through laboratory analysis. The objective of the study was to evaluate the capability of Near -Infrared Reflectance Spectroscopy (NIRS) method in determining tryptophan content of QPM which enhance the efficiency of QPM research efforts by partially replacing more expensive and time-consuming wet chemistry analysis. 268 maize samples were used to develop NIRS models for Tryptophan content. Standard error (SEC) and coefficient of determination for the calibration were 0.007 and 0.76 respectively. When the NIRS model was subjected to external validation with 40 S2 lines from QPM breeding populations, the standard error of prediction (SEP) for validation and coefficient of determination between NIRS and the chemical data were 0.008 and 0.84 respectively. Therefore, from the result it is confirmed that NIRS model is effective tool for screening of QPM from normal maize.
\end{abstract}

Keywords: Quality Protein Maize, Tryptophan, Lysine, NIRS

\section{Introduction}

Maize breeding programs have been focused on increasing stability and yield potential under abiotic and biotic stresses. However, in the past few years, attempts have been made in biofortification programs to increase the nutritional quality of maize for human and animal consumption [1]. Quality protein maize (QPM) is a type of maize with pronounced amounts of two essential amino acids, lysine (Lys) and tryptophan (Trp), and provides increased nutritional value for protein-deficient populations who depend upon maize as a staple food [2].

Multiple genetic systems control and modify the protein quality of QPM; (Gibbon et al., 2005, Krivanek et al., 2007,) therefore, Trp and/or Lys monitoring is required to ensure and maximize genetic gain in breeding programs [4]. Most QPM breeding programs routinely monitor Trp and/or Lys concentrations in protein by colorimetric methods or highperformance liquid chromatography (HPLC) [5]. The colorimetric method is less complex than HPLC but requires $>20 \mathrm{~h}$ to complete analysis because of overnight digestion procedures (Nurit et al., 2009). These chemical analysis methods have limitations include costs, time, and method robustness [2].

Near-infrared reflectance spectroscopy (NIRS) is a technique that combines spectroscopy and mathematics to rapidly produce indirect, quantitative estimates of concentrations of $\mathrm{OH}-, \mathrm{NH}-, \mathrm{CH}-$, or $\mathrm{SH}-$ containing compounds. In comparison to wet chemistry procedures, NIRS requires none or simple sample preparation methods and is a rapid and relatively inexpensive technique that facilitates the analysis of several traits simultaneously [6]. Spectral data are correlated with biochemical components obtained by standard methods. However, NIRS is an indirect method that requires development and validation of calibrations by analysis of a large number of samples covering the range of variability for each trait and with more or less uniform distribution between extreme values [7]. 
Attempts to use NIRS for amino acid quantification in cereals dated to 1978, when Rubenthaler and Bruinsma (1978) successfully developed a NIRS calibration for the Lys content in wheat and barley. Later Fontaine et al., (2002) developed NIRS calibrations for Lys and Trp, among other amino acids, using 258 and 156 temperate maize samples, respectively. The results showed that NIRS data were more accurate than amino acid estimation based on crude protein regression data, but the usefulness of these calibration curves was very limited, as indicated by a ratio of performance deviation (RPD) values lower than 3 , and because the ratios between the standard deviation (SD) of the reference values and the standard error of prediction for the validation curves were not published.

More recently, Aldo et al., (2011) developed NIRS calibrations for protein, lysine and tryptophan content using 276, 756 and 424 maize samples respectively. The samples considered mid altitude and tropical environments of Africa and Mexico. The results showed that NIRS data were accurate enough to screen QPM from normal maize in QPM breeding program.

Even though both lysine and tryptophan concentrations are increased in QPM materials, only tryptophan is analyzed on a routine basis. This is because lysine (Lys) and tryptophan (Trp) values are highly correlated. In addition the colorimetric reaction for lysine determination in maize kernel is time-consuming, and its reproducibility is affected by many factors. This makes it difficult to use it to analyze large numbers of samples; lysine analysis is therefore not recommended for use in a practical breeding program [10].

Breeding programs devoted in developing QPM are being implemented in Ethiopia, and these programs require robust, fast, and inexpensive laboratory methods to screen QPM from normal maize. Currently the colorimetric method with spectrometer used as reference analytical method for determination of Trp in QPM screening. Although various NIRS model developed in different areas, no NIRS model for tryptophan content for QPM screening was developed in Ethiopia.

Our objective, therefore, was to develop and validate NIRS calibration for Tryptophan content based on samples collected from moisture stress, mid altitude and high land QPM breeding program in Ethiopia.

\section{Materials and Methods}

\subsection{Sample Collection and Preparation}

A total of 308 samples from active QPM breeding program, 38 from moisture stress (Melkasa Agricultural Research Center), 137 from Mid altitude (Bako Agricultural Research Center), 63 from High land (Ambo Agricultural Research Center) harvested in 2015 and 30 maize samples with High, Medium and low tryptophan content from Maize Nutrition Quality Laboratory (MNQL), Mexico were used for the development of NIRS calibration. From those 308 samples, 40 samples were selected as validation set for external validation of the developed calibration.

The samples were splitted and half were sent to Maize Nutrition Quality Laboratory (MNQL), Mexico for wet chemistry analysis. The remnant samples were milled using cyclotec mill (Foss Tecator Cyclotec) with $1 \mathrm{~mm}$ sieve and stored in glass cup for NIRS analysis.

\subsection{NIR Analysis}

The milled 2-3 g per sample material was scanned by NIRS monochromator model FOSS 6500 (FOSS NIRSystems, Inc., Silver Spring, Denmark) using small ring cups (internal diameter of $35 \mathrm{~mm}$ and depth of $8 \mathrm{~mm}$ ). Spectra were collected between 400 and $2500 \mathrm{~nm}$, registering the absorbance values $\log (1 / \mathrm{R})$ at $2 \mathrm{~nm}$ intervals for each sample.

\subsection{Wet Chemistry Analysis}

Tryptophan content of the samples was determined at Maize Nutrition Quality Laboratory (MNQL) using colorimetric method described by Nurit et al. (2011). Each 10 samples were included as an internal standard to verify the accuracy of the method.

\subsection{Mathematical Procedures for Calibrations and Validation}

The Calibration equations were developed using WinISI III software from Infrasoft International (FOSS NIR Systems, Inc., Silver Spring, MD). Calibration models were developed using modified partial least-squares (MPLS) regression and cross-validation techniques. Prior to the PLS regression, spectra were pretreated by applying a first and second derivative transformation and MSC Standard, SNV and detrend smoothing techniques. The results of the calibration calculation were monitored by checking the $\mathrm{t}$ outliers with $\mathrm{t}>$ 2.5, GH, and $\mathrm{X}$ outliers $>10$; samples with $\mathrm{t}>2.5$ were deleted from the sample file [11].

The SD between NIRS and reference determinations for the calibration [standard error of calibration (SEC)] and validation sets [standard error of prediction (SEP)] were calculated. And also the coefficient of determination of calibration $\left(\mathrm{R}^{2} \mathrm{c}\right)$ and the coefficient of determination of validation $\left(R^{2} v\right)$ (the fraction of the variance of the reference values explained by the variance of NIRS determinations) were calculated.

In addition, we determined the ratio between the SD and the SEP because the quality and robustness of a NIRS calibration can also be judged by the SEP and SD/ SEP; a $\mathrm{SD} / \mathrm{SEP}$ less than 2 indicates an unsuitable calibration [1].

\section{Results and Discussion}

\subsection{Reference Data and Sample Distribution}

The reference analysis was done at Maize Nutrition Quality Laboratory, CIMMYT, Mexico; using colorimetric method described by Nurit et al. (2011) and the standard 
error for laboratory value using this methodology was found to be 0.04 .

Reference values for samples in the calibration set were distributed over range of $0.02-0.12 \%$, because both normal and QPM maize samples were included; QPM has more than $0.07 \%$ Trp.

\subsection{NIRS Calibration Development}

20 calibrations were developed using modified partial least-squares (MPLS) regression, cross-validation techniques, different data pre-treatment and spectra transformation. From these calibrations three calibration with better calibration statistics were selected for independent validation. NIRS calibration equations, developed on the basis of 242, 236 and 240 samples for cal 1, cal 2 and cal 3 respectively, had high coefficients of determination for calibrations $\left(R^{2} c=0.72\right.$ $0.76)$ and slightly lower coefficients of determination for cross-validations $\left(\mathrm{R}^{2} \mathrm{cv}=0.65-0.69\right)$ (Table 1$)$.

Table 1. Reference Values, NIRS Calibration, and Cross-Validation Statistics for Trp, in the Calibration Set of Maize.

\begin{tabular}{|c|c|c|c|c|c|c|c|c|c|}
\hline Calibrations & Data Pre-treatment & $\mathbf{N}$ & Range & Mean & ${ }^{\mathrm{a}} \mathrm{SD}$ & ${ }^{b} \mathbf{R}^{2} \mathbf{c}$ & ${ }^{\mathrm{c}} \mathrm{SEC}$ & ${ }^{\mathrm{d}} \mathbf{R}^{2} \mathrm{cv}$ & ${ }^{\mathrm{e}} \mathrm{SECV}$ \\
\hline Cal 1 & Standard and MSC & 242 & $0.02-0.10$ & 0.060 & 0.015 & 0.76 & 0.007 & 0.68 & 0.0084 \\
\hline Cal 2 & SNV \& Detrend & 236 & $0.02-0.11$ & 0.060 & 0.015 & 0.74 & 0.007 & 0.69 & 0.0087 \\
\hline Cal 3 & SNV \& Detrend & 240 & $0.02-0.12$ & 0.061 & 0.015 & 0.72 & 0.008 & 0.65 & 0.0090 \\
\hline
\end{tabular}

${ }^{\mathrm{a}} \mathrm{SD}=$ standard deviation. ${ }^{\mathrm{b}} \mathrm{R}^{2} \mathrm{c}=$ coefficient of determination in calibration. ${ }^{\mathrm{c}} \mathrm{SEC}=$ standard error of calibration. ${ }^{\mathrm{d}} \mathrm{R}^{2} \mathrm{cv}=$ coefficient of determination in crossvalidation. ${ }^{\mathrm{e}} \mathrm{SECV}=$ standard error of cross-validation.

And also, the difference between $\mathrm{R}^{2} \mathrm{c}$ and $\mathrm{R}^{2} \mathrm{cv}$ was minor, indicating that the calibrations were homogeneous. The SEC and SECV were small and comparable with the standard error the method which is 0.004 . The difference between SEC (0.007) and SECV (0.0084) of the models were small, that indicate error in prediction of the calibrations for crossvalidation sets. These results were found to be comparable with the result obtained by Aldo et al 2011 at CIMMYT Mexico, Maize Nutrition Quality Laboratory.

\subsection{External/Independent Validation}

An independent validation of the calibrations was performed for Cal 1, Cal 2 and Cal 3 using 40 samples from High land and mid land QPM breeding program in Ethiopia. The coefficients of determination for independent validation ( 2 2 v) were larger than those measured for the cross-validations (Table 2). The SD/SEP ratio except for cal 1 which was 1.9 , both cal 2 and 3 were 2.25 and 2.3 , indicating the calibration is satisfactory because it falls within the range of 2 - 3. Considering both $\mathrm{SD} / \mathrm{SEP}$ ratios and $\mathrm{R}^{2} \mathrm{v}$ values, Cal 2 results show that reliable selection for Tryptophan content is possible by NIRS.

Table 2. Reference Values and External Validation Statistics of the NIRS Calibrations for Tryptophan Contents in Maize.

\begin{tabular}{llllllll}
\hline Calibrations & $\mathbf{N}$ & Range & Mean & SD & $\mathbf{R}_{\mathbf{v}}^{2}$ & SEP & SD/SEP \\
\hline Cal 1 & 40 & $0.033-0.098$ & 0.062 & 0.019 & 0.789 & 0.010 & 1.90 \\
$\mathrm{Cal} 2$ & 40 & $0.033-0.098$ & 0.062 & 0.018 & 0.830 & 0.008 & 2.25 \\
$\mathrm{Cal}$ 3 & 40 & $0.033-0.098$ & 0.062 & 0.018 & 0.790 & 0.008 & 2.31 \\
\hline
\end{tabular}

$\mathrm{SD}=$ standard deviation. $\mathrm{R}^{2} \mathrm{v}=$ coefficient of determination in for validation. $\mathrm{SEP}=$ standard error of prediction.

$\mathrm{SD} / \mathrm{SEP}$ for validation of the present calibration was 2.2 which is comparable with the result obtained by Aldo Rosales et al 2011 that is 2.6. Samples used to develop the calibrations included a wide range of material from low land, mid-altitude and high land research areas of to ensure broad applicability of the NIRS models.

\section{Conclusion}

Overall performance of the developed calibration indicates that NIRS can be confidently used and offer a fast and simple screening option for thousands of samples that must be evaluated every cycle because extractions and chemical reactions are not required. It is recommended to verify the accuracy of extreme values by chemical analysis, especially for very advanced breeding material.

\section{References}

[1] Montes, J.; Utz, H.; Schipprack, W.; Kusterer, B.; Muminovic, J.; Paul, C.; Melchinger, A. E.: Near- infrared spectroscopy on combine harvesters to measure maize grain dry matter content and quality parameters. Plant Breed. 125, 2006. 591-595.

[2] Aldo R, Luis G., Ezequiel O., Catalina I., and Natalia P.; NearInfrared Reflectance Spectroscopy (NIRS) for Protein, Tryptophan, and Lysine Evaluation in Quality Protein Maize (QPM) Breeding Programs. J. Agric. Food Chem., 59, 2011, 10781-10786.

[3] Gibbon, B.; Larkins, B.: Molecular genetics approaches to developing quality protein maize. Trends Genet. 21, 2005, 227-233. 
[4] Krivanek, A. F.; De Groote, H.; Gunaratna, N.; Diallo, A.; Friesen, D.: Breeding and disseminating quality protein maize (QPM) for Africa. Afr. J. Biotechnol., 6, 2007. 312-324.

[5] Nurit, E.; Tiessen, A.; Pixley, K.; Palacios-Rojas, N.: A reliable and inexpensive colorimetric method for determining protein-bound tryptophan in maize kernels. J. Agric. Food Chem. 57, 2009. 7233-7238.

[6] Melchinger, A. E.; Schmidt, G.; Geiger, H.: Evaluation of near infra-red reflectance spectroscopy for predicting grain and stover quality traits in Maize. Plant Breed. 97, 1986. 20-29.

[7] Shenk, J.; Westerhaus, M.: Population definition, selection, and calibration procedures for near infrared reflectance spectroscopy. Crop Sci. 31, 1991. 469-474.
[8] Rubenthaler, G. L.; Bruinsma, B. L.: Lysine estimation in cereals by NIR. Crop Sci. 18, 1978. 1039-1042.

[9] Fontaine, J.; Schirmer, B.; Horr, J.: Near-infrared reflectance spectroscopy (NIRS) enables the fast and accurate prediction of essential amino acid contents. J. Agric. Food Chem., 50, 2002, 3902-3911.

[10] Vivek B. S., A. F. Krivanek, N. Palacios-Rojas, S. TwumasiAfriyie, and A. O. Diallo.: Breeding Quality Protein Maize (QPM): Protocols for Developing QPM Cultivars. Mexico, D. F.: CIMMYT, 2008. 105.

[11] Zum Felde, T.; Baumert, A.; Strack, D.; Becker, H.; Moellers, C.: Genetic variation for sinapate ester content in winter rapeseed (Brassica napus L.) and development of NIRS calibration equations. Plant Breed. 126, 2007, 291-296. 